\title{
TIC Y EDUCACIÓN. Usos de Google Plus en el proceso de enseñanza - aprendizaje
}

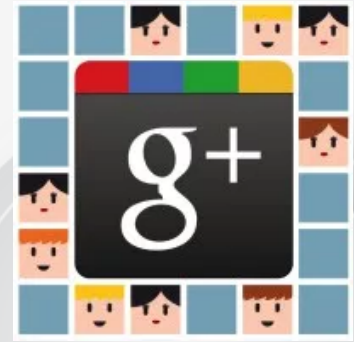

\section{RESUMEN}

El artículo presenta los resultados de la investigación "usos de la red social Google Plus, en una experiencia de enseñanza y aprendizaje de educación universitaria"; el enfoque fue interpretativo, basado en un estudio de caso simple.

Los hallazgos mostraron que Google Plus se utilizó principalmente con fines comunicativos entre el profesor y el estudiante. Las interacciones que emergieron alrededor de los espacios que proporciona, tales como chats, comunidades, el mensajero, los hangouts, entre otros, estuvieron relacionadas específicamente con explicaciones, recordatorios, indicaciones, recomendaciones, acuerdos y la motivación; otras enfatizaron en la expresión de sentimientos, emociones, aficiones e intereses.

Palabras clave: TIC; Google Plus; enseñanza; aprendizaje; educomunicación.

Recibido. Febrero 08, 2017 Aceptado. Agosto 14, 2017

**Magíster en Educación. Docente, Universidad Tecnológica de Pereira.

Orcid: https://orcid.org/0000-0001-8657-6067

Email:kagutierrez@utp.edu.co 


\title{
EDUCATION AND ICT.
}

\section{Uses of Google Plus in teaching - learning process}

\begin{abstract}
Summary
The general purpose of this research was to understand the "actual uses of the social network called Google Plus, in a teaching-learning experience at the University level", its interpretative focus, based on a simple case study.

The uses established the existing relationships between what the teacher prepares and what is carried out. Findings show that, regarding the planning and execution of this experience, Google Plus was used mainly with communicative purposes between the teacher and the student. However, during execution, such interactions emerged in other spaces. Finally, it is concluded that it is necessary to continue structuring educational projects through social networking sites like Google Plus, using its applications with pedagogical and didactic intentions.
\end{abstract}

Keywords: ITC; Google Plus; teaching; learning.

Received. February 8, 2017

Accepted. August 14, 2017

TIC E EDUCAÇÃO

Usos de Google Plus no processo de educação - aprendizagem

\begin{abstract}
Resumo
O artigo apresenta os resultados da investigação "usos da rede social Google Plus, numa experiência de educação e aprendizagem da educação universitária"; o foco foi interpretativo, baseado num estudo de caso simples.

As descobertas mostraram que Google Plus, era principalmente usado com fins comunicativos, entre o professor e o estudante. As interações que emergiram ao redor dos espaços que proporciona, tais como conversas, as comunidades, o mensageiro, os hangouts, entre outros, estiveram relacionados específicamente com explicações, lembranças, indicações, recomendações, acordos e a motivação. Outros enfatizaram na expressão de sentimentos, emoções, preferências e interesses.
\end{abstract}

Palavras chaves: TIC; Google Plus; ensino; aprendizagem; educomunicação.

Recebido. Fevereiro 8, 2017

Aceitado. Agosto 14, 2017. 


\section{INTRODUCCIÓN}

$[$ E uso creciente y masivo de las TIC (Tecnologías de la Información y la Comunicación), en la sociedad, ha hecho que se generen procesos de transformación social, cultural, política y económica; sin embargo, los escenarios educativos parecen ser un campo que va a un ritmo más lento, en cuanto a la incorporación de este tipo de tecnologías, así lo plantea Martínez (2012) cuando afirma que, "la escuela sigue avanzando poco en el uso educativo y didáctico de los medios informáticos" (p. 24). Si los cambios que se generan en torno a las TIC, son interpretados y además implementados por esferas organizacionales, gubernamentales, comunicativas, entre otras, habría que cuestionarse alrededor de las razones por las cuales, los sistemas educativos, han tenido dificultad para incorporarlas asertivamente y potenciar sus prácticas con las posibilidades transformadoras que estas ofrecen.

Los estudios prácticos que se centran en la observación, el análisis y la implementación de las TIC en las instituciones educativas son aún limitados. López de la Madrid (2013) asegura que "el uso de este tipo de tecnologías en las prácticas educativas se ha focalizado en un instrumentalismo tecnológico, dejando a un lado las posibilidades transformadoras e innovadoras que ofrecen" (p.54).

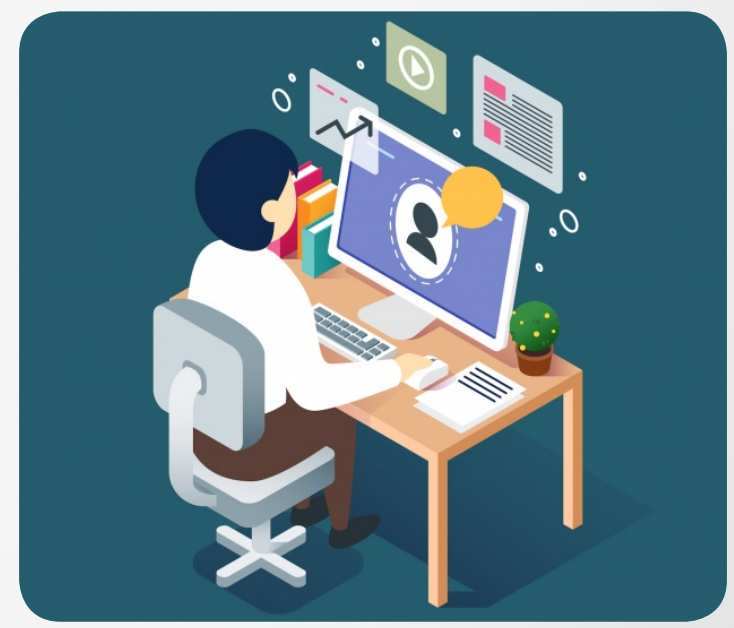

Si se vive en una nueva sociedad caracterizada por las transformaciones que generan las TIC, por qué la escuela no incorpora este tipo de tecnologías, ese es uno de los cuestionamientos que también se plantean organismos internacionales como la UNESCO (2012), que busca desarrollar nuevas estrategias para que, en las instituciones educativas de los estados miembros, se logre una alfabetización en información, que permita a su vez un mayor énfasis en la formación de profesorado. Muestra de esto se observa en sus publicaciones: Media literacy and new humanism (2012) y Pedagogies of media and information lietracies (2010). Se requiere el desarrollo de actividades que permitan la comprensión de los contenidos temáticos, actitudinales y procedimentales que se generan alrededor de las TIC.

El Ministerio de Educación en alianza con el Ministerio de TIC de Colombia, han impulsado programas como "Computadores para Educar" creado desde el año 2001, el cual busca promover "el acceso, el uso y el aprovechamiento de las tecnologías de la información y la comunicación en las comunidades educativas" (Computadores para Educar, 2004), incluyendo mayoritariamente a la población rural. Esta apuesta está acompañada de otros proyectos estatales como "Colombia digital", "Vive digital" y "Educa digital", que centralizan sus objetivos en la profundización, aprendizaje y apropiación de las TIC.

En Risaralda, "Computadores para Educar" dio inicio en el 2011, a cargo de la Universidad Tecnológica de Pereira quien fue la encargada de "formar a 266 gestores de las TIC para los departamentos de Caldas, Quindío, Valle y Risaralda, de estos, 98 eran de Risaralda" (MEN, 2011), se instruyó a los profesores en el manejo de los computadores y su uso en las prácticas didácticas.

Autores como González, D., Bermeo, H. \& Ortiz, G. (2011):

Consideran que los estudiantes
universitarios de las últimas
generaciones como nativos digitales,
requieren mucho más que antes
de ambientes de aprendizaje que
fomenten la creación de nuevo
conocimiento y competencias a
través de una mayor interacción,
integración, colaboración no solo con
sus compañeros y profesores, sino
con todo actor en su entorno físico
y digital que pueda proveerles de
información y posterior conocimiento.
(p.54)

Las necesidades y retos de la sociedad en relación con la incorporación de las TIC en procesos educativos haciendo uso de las redes sociales, permiten plantearse la siguiente pregunta de investigación: ¿Qué usos de la red social Google Plus se desarrollan en una experiencia de enseñanza y aprendizaje universitaria? 


\section{Metodología}

Tipo de investigación. El diseño de esta investigación se enmarcó en el enfoque interpretativo. Para Erickson (1986) este trata de comprender la realidad en su carácter específico, distintivo y particular, adicional se ofrece una mirada socioconstructivista, que asume el proceso educativo del aula desde las interrelaciones de los componentes del triángulo interactivo, planteado por Coll \& Monereo (2008), como las relaciones mutuas que se dan entre el estudiante, contenido y profesor; a partir de la práctica educativa constituida en el núcleo básico de los procesos formales de enseñanza y aprendizaje.

Estrategia metodológica. Se abordó el estudio de casos simples, planteado por Yin (1998) y Stake (1999), en búsqueda de la comprensión de los fenómenos sociales que parten de la misma fundamentación teórica en la unidad de observación y análisis, que son propuestas didácticas completas y entre ellas se busca una variación máxima atendiendo aspectos relevantes en cuanto al uso de las redes sociales, tales como el carácter bimodal, los contenidos de enseñanza y aprendizaje, así como la diversidad y riqueza de las estrategias y herramientas incorporadas (trabajo individual, grupal, comunidades, etc.).

Unidad didáctica de análisis de datos cuantitativos. La investigación se desarrolló mediante una experiencia de enseñanza y aprendizaje de "análisis de datos cuantitativos" dentro de la asignatura Informática educativa I, que hace parte del plan de estudios de VII semestre de la Licenciatura en Pedagogía Infantil, en un grupo conformado por 20 estudiantes, todas mujeres.

La unidad didáctica de esta experiencia se diseñó a través de la metodología TPACK, la cual consiste en la articulación de 3 tipos de conocimiento: tecnológico, pedagógico y disciplinar. Esta es una forma de abordar el proceso de enseñanza - aprendizaje en la cual se busca realizar una integración asertiva de las TIC y las lógicas educativas.

Instrumentos: Se realizó una entrevista semiestructurada inicial y final, la cual es concebida por Blasco y Otero (2008) como una "técnica en la que las preguntas están definidas previamente, en un guion, pero la secuencia, así como su formulación, pueden variar en función de cada sujeto entrevistado" (p.48), esta se utilizó para contrastar lo que el docente pensaba, planeaba y ejecutaba con respecto a los usos pedagógicos de las redes sociales.

Para abordar a los estudiantes se aplicaron dos cuestionarios; uno centrado en explorar las expectativas iniciales de estos frente a la experiencia de enseñanza y aprendizaje mediada a través de las redes sociales, dicho formulario se aplicó al inicio y al final del proceso. El otro cuestionario se centró en los conocimientos previos, mediante los cuales se quiso conocer si los estudiantes tenían la información básica en cuanto al uso y manejo de los temas que se requerían para desarrollar la unidad.

Categorías, criterios y características de uso. Se estableció una matriz categorial que definiera ciertos usos de Google Plus, en relación a los procesos de enseñanza y aprendizaje; posibilitando así su descripción y posterior interpretación. En la revisión del tema de las TIC y la educación, se llegó a Coll, Maury y Onrubia (2008), como apoyo teórico y bibliográfico, estos autores llevaron a cabo un sistema de clasificación de usos de estas tecnologías, intentando superar limitaciones que pueden llegar a coartar el estudio y el análisis de las posibilidades de este tipo de tecnologías.

Las categorías que hicieron parte de la matriz adaptada para efectos de la investigación fueron las siguientes:

- Google Plus como instrumento mediador entre los estudiantes y los contenidos.

- Google Plus como instrumento mediador entre el profesor y los contenidos.

- Google Plus como instrumento mediador entre el profesor y estudiantes o entre los estudiantes.

- Google Plus como instrumento mediador de la actividad conjunta desplegada en la realización de la tarea.

- Google Plus como instrumento configurador de espacios de trabajo. 


\section{Resultados y discusión}

\subsection{Identificación y descripción de usos planeados}

La identificación y descripción de los usos planeados fue el primer propósito investigativo; desde la perspectiva de Coll et al. (2008), son aquellos potenciales y previstos que surgen en un primer momento de planeación de la experiencia educativa.

En la tabla 1 se muestra los usos que se presentaron en la fase de la planeación de la experiencia y los porcentajes que les corresponden, los cuales fueron analizados en detalle.

\section{Tabla 1. Usos planeados de Google Plus}

\begin{tabular}{|c|c|c|c|c|}
\hline Categorías de uso & Criterios de uso & $\begin{array}{l}\text { Total } \\
\text { sub. }\end{array}$ & $\%$ sub. & Total cat. \\
\hline \multirow{3}{*}{$\begin{array}{l}\text { Google Plus como } \\
\text { instrumento mediador } \\
\text { entre los estudiantes y } \\
\text { los contenidos }\end{array}$} & Aplicaciones digitales & 46 & $53 \%$ & \multirow[t]{3}{*}{86} \\
\hline & Acceso a contenidos de enseñanza y aprendizaje & 18 & $21 \%$ & \\
\hline & Realización de tareas y actividades de aprendizaje & 22 & $26 \%$ & \\
\hline \multirow{3}{*}{$\begin{array}{l}\text { Google Plus como } \\
\text { instrumento mediador } \\
\text { entre el profesor y el } \\
\text { contenido }\end{array}$} & Gestión de contenidos & 7 & $37 \%$ & \multirow[t]{3}{*}{55} \\
\hline & Planificación y preparación de actividades & 35 & $45 \%$ & \\
\hline & Registro de actividades & 13 & $18 \%$ & \\
\hline \multirow{4}{*}{$\begin{array}{l}\text { Google Plus como } \\
\text { instrumento mediador } \\
\text { entre elbprofesor y } \\
\text { estudiantes o entre los } \\
\text { estudiantes }\end{array}$} & $\begin{array}{c}\text { Intercambios comunicativos entre el profesor y } \\
\text { estudiantes no relacionados con el contenido }\end{array}$ & 12 & $24 \%$ & \multirow[t]{4}{*}{89} \\
\hline & $\begin{array}{c}\text { Intercambios comunicativos entre el profesor y } \\
\text { estudiantes relacionados con el contenido }\end{array}$ & 59 & $57 \%$ & \\
\hline & $\begin{array}{c}\text { Intercambios comunicativos entre estudiantes no } \\
\text { relacionados con el contenido }\end{array}$ & 9 & $10 \%$ & \\
\hline & $\begin{array}{c}\text { Intercambios comunicativos entre estudiantes } \\
\text { relacionados con el contenido }\end{array}$ & 9 & $9 \%$ & \\
\hline \multirow{4}{*}{$\begin{array}{l}\text { Google Plus como } \\
\text { instrumento mediador de } \\
\text { la actividad conjunta } \\
\text { desplegada en la tarea }\end{array}$} & \begin{tabular}{|ll} 
Auxiliares & $\begin{array}{l}\text { amplificadores determinadas } \\
\text { actuaciones del profesor }\end{array}$
\end{tabular} & 11 & $22 \%$ & \multirow[t]{4}{*}{49} \\
\hline & $\begin{array}{|ccc|}\text { Auxiliares } & 0 & \text { amplificadores determinadas } \\
& \text { actuaciones del estudiante } \\
\end{array}$ & 19 & $39 \%$ & \\
\hline & Realizar seguimiento & 5 & $10 \%$ & \\
\hline & $\begin{array}{l}\text { Apoyo para el desarrollo de las actividades y de los } \\
\text { productos }\end{array}$ & 14 & $29 \%$ & \\
\hline \multirow{4}{*}{$\begin{array}{l}\text { Google Plus como } \\
\text { instrumento configurador } \\
\text { espacios de trabajo }\end{array}$} & $\begin{array}{c}\text { Configuración de espacios de aprendizaje individual } \\
\text { en línea }\end{array}$ & 9 & $24 \%$ & \multirow[t]{3}{*}{37} \\
\hline & \begin{tabular}{|cc} 
Configuración de espacios de aprendizaje \\
colaborativo en línea
\end{tabular} & 10 & $27 \%$ & \\
\hline & Configuración de espacios en aprendizaje en línea & 18 & $49 \%$ & \\
\hline & Total & 316 & & 316 \\
\hline
\end{tabular}


Para la identificación y descripción de los usos planeados, se analizó en primera instancia cada uno de los aspectos evidenciados en la unidad didáctica; su enfoque, objetivos, contenidos, metodología, evaluación, entre otros elementos. Con respecto a la metodología, es en este punto en el que se hace observable el TPACK, como por ejemplo en la realización de los videos tutoriales, en los que se debía reflejar un conocimiento disciplinar alrededor del tema del análisis de datos cuantitativos, el conocimiento pedagógico y didáctico al momento de su elaboración; teniendo en cuenta la población a la que se planeaba orientar el tópico y la transposición necesaria para abordar educativamente el tema. Por último, se evidencia el manejo del editor de video en el cual se puso en juego el componente tecnológico. Esta simbiosis entre disciplina, pedagogía y tecnología fue pensada en cada una de las actividades de la unidad didáctica.

Por otro lado, se observaron los instrumentos de recolecciónaplicadosinicialmente; elcuestionario de conocimientos previos y de expectativas dirigido a los estudiantes y la entrevista realizada al docente. En lo que respecta al cuestionario de conocimientos previos se constató que existe un manejo básico de internet y redes sociales, pero que en lo que respecta a las hojas de cálculo se requería, en ese momento, de un mayor acompañamiento. Alrededor del cuestionario de expectativas iniciales los estudiantes detallaron su experiencia al interior de las redes sociales con fines centrados en el entretenimiento, motivo por el cual manifestaron curiosidad en torno a su uso educativo y más específicamente afirmaban desconocer cómo a través de este espacio se trabajaría el análisis cuantitativo de datos y cómo se llevaría a cabo la relación con el docente.

El docente manifestó que esperaba que la red social se convirtiera en un verdadero ambiente de enseñanza y aprendizaje que proporcionara todas las herramientas para trabajar de manera significativa el análisis de datos cuantitativos con sus estudiantes, puesto que sus experiencias anteriores en el uso de tecnologías de la información y de la comunicación le habian ofrecido otra perspectiva a sus clases en términos comunicativos, pero esta resultaba retadora debido a que involucraba una inmersión total en un escenario virtual como el configurado por Google Plus y su preocupación se centraba en la manera en la que se desarrollaría su relación con los estudiantes.
Teniendo en cuenta lo anterior y los aspectos expuestos en la tabla 1, en términos generales, la relación entre profesores y estudiantes fue el componente fuerte y que más se tuvo en cuenta al momento de considerar las actividades de enseñanza, aprendizaje y las interacciones que emergieron; relacionadas demaneradirectacomo explicaciones, recordatorios, indicaciones, recomendaciones y acuerdos; y la motivación a los estudiantes. indirecta y meramente formal como la solicitud de información y presentación personal, saludos, despedidas, expresión de sentimientos, emociones, aficiones e intereses. Dichas interacciones, se planearon en los espacios comunicativos que proporciona Google Plus, tales como los chats, las comunidades, el mensajero, los hangouts, entre otros.

Tras el análisis de la planeación de la experiencia de enseñanza y aprendizaje y los usos planeados derivados de la matriz categorial expuesta en un inicio, el paso a seguir, consistió en el análisis de las sesiones que se llevaron a cabo para abordar el segundo objetivo de la investigación, que fue la identificación y descripción de los usos ejecutados, para lo cual se realizó una lectura y análisis de los datos provenientes de los registros digitales que dieran cuenta de lo que sucedía en cada sesión al interior de la red social.

Los usos ejecutados, son aquellos que se presentan en el transcurso de la experiencia de enseñanza y aprendizaje, al final de la misma. Analizarlos permitió hallar las dimensiones fundamentales de las prácticas educativas, como lo afirma Coll et al. (2008), consiguiendo con ello en primera medida confirmar la veracidad de tales usos y obtener una amplia selección de aspectos que cobran relevancia o no al momento de implementar las tecnologías de la información y la comunicación en escenarios educativos. Para esta segunda fase también se tuvo en cuenta la matriz categorial expuesta inicialmente: 


\section{Tabla 2. Usos ejecutados de Google Plus}

\begin{tabular}{|c|c|c|c|c|}
\hline Categorías de uso & Criterios de uso & $\begin{array}{c}\text { Total } \\
\text { sub. }\end{array}$ & $\%$ sub. & $\begin{array}{l}\text { Total } \\
\text { cat. }\end{array}$ \\
\hline \multirow{3}{*}{$\begin{array}{l}\text { Redes sociales como } \\
\text { instrumentos mediadores } \\
\text { entre los estudiantes y los } \\
\text { contenido }\end{array}$} & Aplicaciones digitales & 71 & $63 \%$ & 112 \\
\hline & Acceso a contenidos de enseñanza y aprendizaje & 17 & $15 \%$ & \\
\hline & Realización de tareas y actividades de aprendizaje & 24 & $22 \%$ & \\
\hline \multirow{3}{*}{$\begin{array}{l}\text { Redes sociales como instrumentos } \\
\text { mediadores entre el profesor y el } \\
\text { contenido }\end{array}$} & Gestión de contenidos & 57 & $45 \%$ & 134 \\
\hline & Planificación y preparación de actividades & 51 & $37 \%$ & \\
\hline & Registro de actividades & 26 & $18 \%$ & \\
\hline \multirow{4}{*}{$\begin{array}{l}\text { Redes sociales como instrumentos } \\
\text { mediadores entre el profesor y } \\
\text { estudiantes o entre los estudiantes }\end{array}$} & $\begin{array}{c}\text { Intercambios comunicativos entre el profesor y estudiantes no } \\
\text { relacionados con el contenido }\end{array}$ & 46 & $24 \%$ & 195 \\
\hline & $\begin{array}{c}\text { Intercambios comunicativos entre el profesor y estudiantes } \\
\text { relacionados con el contenido }\end{array}$ & 111 & $57 \%$ & \\
\hline & $\begin{array}{l}\text { Intercambios comunicativos entre estudiantes no relacionados } \\
\text { con el contenido }\end{array}$ & 20 & $10 \%$ & \\
\hline & $\begin{array}{l}\text { Intercambios comunicativos entre estudiantes relacionados con } \\
\text { el contenido }\end{array}$ & 18 & $9 \%$ & \\
\hline \multirow{4}{*}{$\begin{array}{c}\text { Redes sociales como instrumentos } \\
\text { mediadores de la actividad conjunta } \\
\text { desplegada en la tarea }\end{array}$} & $\begin{array}{c}\text { Auxiliares o amplificadores determinadas actuaciones del } \\
\text { profesor }\end{array}$ & 17 & $14 \%$ & 123 \\
\hline & $\begin{array}{c}\text { Auxiliares o amplificadores determinadas actuaciones del } \\
\text { estudiante }\end{array}$ & 20 & $16 \%$ & \\
\hline & Realizar seguimiento & 13 & $11 \%$ & \\
\hline & Apoyo para el desarrollo de las actividades y de los productos & 73 & $59 \%$ & \\
\hline \multirow{3}{*}{$\begin{array}{l}\text { Redes sociales como instrumentos } \\
\text { configuradores de espacios de trabajo }\end{array}$} & Configuración de espacios de aprendizaje individual en línea & 28 & $30 \%$ & 94 \\
\hline & Configuración de espacios de aprendizaje colaborativo en línea & 21 & $22 \%$ & \\
\hline & Configuración de espacios de actividad en línea & 45 & $48 \%$ & \\
\hline & Total & 658 & 658 & \\
\hline
\end{tabular}

Fuente: elaboración propia basada en el corpus documental

En la ejecución de la experiencia de enseñanza y aprendizaje, sesión tras sesión de manera síncrona y asíncrona, de acuerdo a cada uno de los contenidos que estructuraban la unidad didáctica de análisis de datos cuantitativos (tablas de distribución de frecuencia, medidas de tendencia central, medidas de dispersión y representación de gráficos estadísticos), los estudiantes, como usuarios activos de la internet que hacen parte de la sociedad red planteada por Castells (2000), asumieron ese rol activo, dinámico y continuo, característico de los miembros de este tipo de sociedad al interactuar y ser protagonistas de la realización de sus tareas de aprendizaje e inclusive ofrecieron su ayuda para resolver inquietudes a sus pares.

Por su parte el docente en esta experiencia asumió varias de las funciones expuestas por Cabero (2001) como por ejemplo ser facilitador de información y del aprendizaje, además de diseñador de medios, puesto que a través de Google Drive y sus posibilidades ofimáticas elaboró documentos de apoyo como hojas de cálculo y presentaciones que eran compartidas a través del muro de Google Plus, espacio en el que a su vez se compartía material audiovisual importante para el desarrollo de la experiencia como los videos tutoriales, siendo el creador de cada una de estas ayudas. Por otro lado, asumió una figura de moderación, tutoría y orientación que se vio reflejada en los espacios comunicativos propios de Google Plus, como los chats, los hangouts y las publicaciones registradas en el espacio de la red. En estas se mostraba un seguimiento y resolución a cada uno de los interrogantes, sugerencias y peticiones que tenían los estudiantes. 
A raíz de lo expuesto anteriormente se encuentra que, en el transcurso de la unidad primó el intercambio comunicativo entre profesores y estudiantes,lo que se vio plasmado en el espacio configurado por Google Plus y sus aplicaciones, en las cuales sobresalieron aquellas interacciones que estaban específicamente relacionadas con los contenidos 0 tareas de aprendizaje, tales como la presentación de la unidad didáctica y las actividades, la explicación de las mismas, las recomendaciones y los acuerdos.

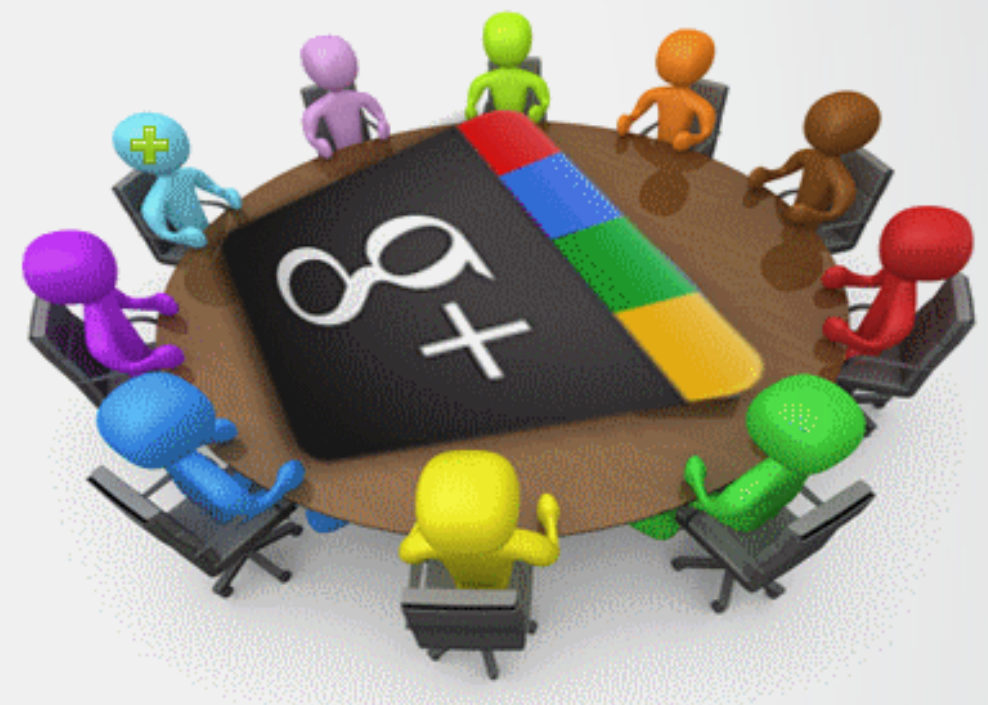

A su vez, se observaron representaciones importantes centradas en las relaciones que se establecieron entre estudiantes - contenido y profesor - contenido, en lo que respecta a la primera, el estudiante en la ejecución hace uso de aplicaciones para subir y visualizar videos, almacenar y crear archivos en línea, buscar y seleccionar información, accede a repositorios de contenidos multimedia y desarrollar procesos de autoaprendizaje.

En la relación profesor - contenido, se realizó la búsqueda, selección y organización de información; el acceso a repositorios de tareas de aprendizaje, programación, realización de contenidos multimedia, registro, seguimiento de la participación y de los productos de los estudiantes.

\subsection{Contrastación de usos planteados y ejecutados para hallar}

\section{los reales}

Para facilitar dicho contraste se utilizó una función a través de una hoja de cálculo que permitió evidenciar los usos reales de Google Plus. Esta clasificación se tomó de la investigación realizada por Coll et al. (2008), titulada Análisis de los usos reales de las TIC en contextos educativos formales: una aproximación sociocultural.

En términos generales, la mayoría de los usos planeados fueron ejecutados, sin embargo, hay diferencias, es decir, que al momento de realizar las actividades, surgió un aumento o una disminución frente a lo que se tenía planeado, estos detalles serán descritos a continuación, pero antes, se hace énfasis en que, para observar esas divergencias, se estableció una diferencia entre la frecuencia de los usos planeados y desarrollados.

Al finalizar esta contrastación, se encuentra que hay equivalencia entre las categorías que más y menos participación tuvieron tanto en la planeación como en la ejecución y que corresponden, en ese orden de ideas a la categoría 3: Google Plus como instrumento mediador entre el profesor y estudiantes 0 entre los estudiantes y la 5: Google Plus como instrumento configuradores de espacios de trabajo.

También se encontraron, usos planeados ejecutados; planeados no ejecutados y usos no planeados que fueron ejecutados, hecho que coincide en los resultados hallados por Coll et al. (2008).

\footnotetext{
Usos previstos de las TIC que no aparecen en el desarrollo real de las secuencias, usos no previstos que sí aparecen, usos previstos como prioritarios que acaban teniendo un papel secundario, usos previstos como secundarios que acaban siendo los más utilizados realmente. (Coll et al, 2008, p. 80)
}

A esta conclusión llegó Coll et al. (2008) tras la aplicación de 5 secuencias didácticas, a través de las cuales se identificó los usos reales que se condensan en la cita anteriormente descrita y que coinciden con los hallados tras la aplicación de la experiencia educativa de esta investigación. 
Dentro de los usos planeados y ejecutados se pudieron detectar usos concretos, innovadores y transformadores, pero también usos que como bien lo afirma Coll et al. (2008) no aportan ningún valor significativo a los procesos de enseñanza y aprendizaje; el impacto positivo o neutral de estos se pueden detectar en la frecuencia con la que se produjeron.

Con el análisis e interpretación realizados se estableció tal y como lo afirman Coll et al. (2008), que no es pertinente un "determinismo pedagógico" alrededor de los usos elaborados por él, y que fueron adaptados para esta investigación; ninguna de las categorías es más innovadora o transformadora que otra. Se encontraron usos que no comportan mayores procesos de cambio, ni novedad frente a las prácticas tradicionales, como la presentación y solicitud de información; programación de actividades y acceso a los repositorios de contenidos.

Sin embargo, sí se puede afirmar desde la lógica del mismo autor, que para que la potencialidad de las redes sociales influya en los procesos inter e intra-psicológicos que se encuentran en los procesos de enseñanza y aprendizaje, es necesario pensar en una "actividad conjunta en torno a los contenidos" y esta se presenta con mayor énfasis en las categorías: redes sociales como instrumentos mediadores de la actividad conjunta desplegada en la tarea y $y$ de estas como instrumentos configuradores de espacios de trabajo, en las que se espera una mayor manifestación de las potencialidades educativas de las TIC.

\section{CONCLUSIONES}

El proceso de comprensión de los usos reales de Google Plus, fue estructurado a través de una matriz categorial que no fue fácil de construir, pero que, sin embargo, adaptando los postulados de Coll, Onrubia y Onrubia (2007), permitió observar "las formas de organización de la actividad conjunta" (p. 24), que fueron entendidas como aquellos usos que se generan en una experiencia educativa, producto de las relaciones estudiantes-contenidos, profesores-contenidos, profesores-estudiantes y estudiantes-estudiantes. Interacciones que surgen alrededor del triángulo interactivo y que abordadas de manera integral, en este proceso investigativo, contribuyeron al estudio de la incorporación de las redes sociales, específicamente Google Plus, en los procesos educativos.

Sin embargo, y como bien lo expone Coll et al. (2008) tras la clasificación, de usos de las TIC, este tipo de categorizaciones están expuestas a una constante revisión y a un continuo ajuste, razón por la cual, es necesario seguir explorando en la construcción de una tipología de usos de las redes sociales. En este caso, de acuerdo a las necesidades del contexto en el cual son utilizadas, teniendo en cuenta la globalidad que encierra el hecho educativo y colocando el énfasis en aquellos usos "que puedan ayudarnos a poner en relación la actividad que se desarrolla en el aula y los resultados de aprendizaje finalmente alcanzados" (Coll et al., 2008, p.24), con el fin de potenciar las experiencias educativas.

Las diferencias entre las expectativas que se consignan en las apuestas teóricas, y lo que realmente pasa en los ejercicios prácticos, es un hecho que se reflejó de igual manera en esta experiencia educativa, ya que, los estudiantes que hicieron parte de la investigación no tenían un mayor conocimiento de la red social Google Plus, por lo que se presentaron dificultades frente al manejo técnico de las herramientas y aplicaciones, a las que tenían acceso, lo cual se vio reflejado en la confusión que se manifestó a través del cuestionario que fue aplicado al final de la experiencia.

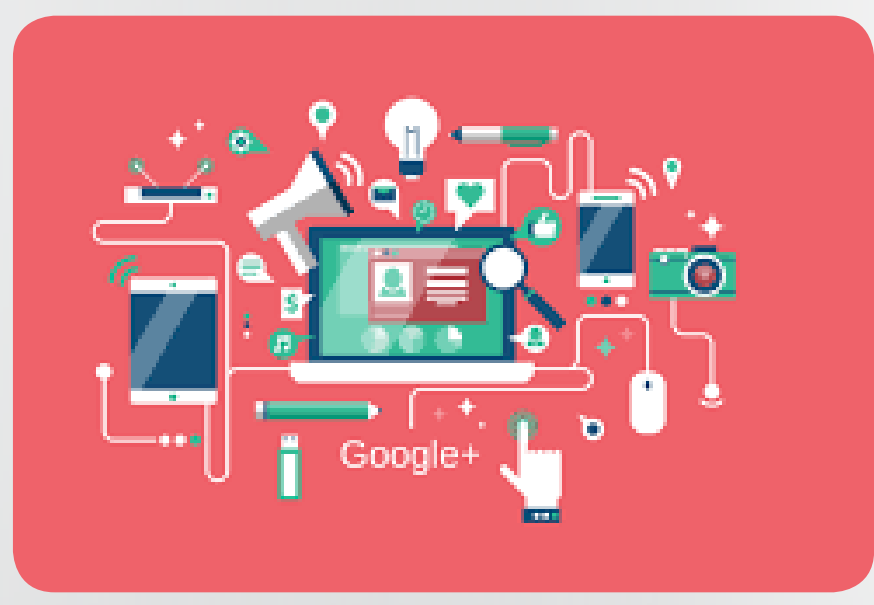

De acuerdo a lo anterior y basándose en las especificidades y vivencias que se tuvieron a lo largo de la experiencia, se recomienda para futuras indagaciones en redes sociales y de la virtualidad como tal, ahondar en la resignificación de los roles, tanto el del profesor como de los estudiantes y el contenido. El primero debe ser estratégico cuando se desenvuelve en este tipo de espacios, recurriendo a la practicidad 
de las herramientas que existen; pero antes de incurrir en la virtualidad, resulta pertinente un acompañamiento presencial, especialmente para orientar los procedimientos básicos, precisos para el manejo de las aplicaciones.

Por otro lado, los estudiantes deben ser conscientes de que, en este tipo de experiencias, factores como la disciplina, la autorregulación y la automotivación son indispensables, puesto que los estudiantes deciden cuándo y cómo abordar los contenidos que se presentan en la modalidad virtual. Por último, los contenidos deben ser presentados de otra manera, la multimedia y el hipertexto, hacen que la linealidad de los textos no sea muy bien recibida cuando se trabaja en torno a las tecnologías digitales.

Esta investigación buscó aportar al estudio de la incorporación de las TIC a los procesos de enseñanza y aprendizaje, desde la planeación, la ejecución y el contrataste de los usos de la red social Google Plus, razón por la cual, para finalizar, se recomienda seguir explorando el uso de esta red social como un espacio en el que se pueden configurar procesos de enseñanza y aprendizaje más allá del aula de clase.

La utilización de Google Plus en otros contextos en los que se tenga un interés de alfabetización desde diversas lógicas, puesto que su potencial comunicativo se configura como una poderosa herramienta para procesos educativos de diversa índole. Se requiere una revisión constante, una evaluación integral y una retroalimentación de las propuestas educativas, en las cuales se tengan en cuenta las interrelaciones que se presentan entre los actores, transformar e innovar las prácticas educativas.

Las instituciones de educación superior deben extender sus procesos de formación a otros ámbitos donde los estudiantes puedan adquirir competencias para un aprendizaje continuo, lo que requiere que los escenarios educativos formales no se asuman como centro sino como uno de los nodos de ese entramado en que los educandos se desenvuelven de manera espontánea; se refleje la creación y promoción de experiencias que le apunten a la innovación y la transformación. (Salinas, 2004).

\section{BIBLIOGRAFÍA}

Blasco, T., \& Otero, L. (2008). Técnicas conversacionales para la recogida de datos en investigación cualitativa: La entrevista. Nure Investigación, 33. Disponible en: http:// www.nureinvestigacion.es/FICHEROS ADMINISTRADOR/F_METODOLOGICA/ formet_332622008133517.pdf

Cabero, J. (1992b). Los medios en los centros de enseñanza: la experiencia española, en CMIDE: Cultura, educación y comunicación, Sevilla: Ayuntamiento de Sevilla, 65-76

Castells, M. (2000). Internet y la sociedad red. Lección inaugural del programa de doctorado sobre la sociedad de la información y el conocimiento. Universitat Oberta de Catalunya. Obtenido de http:// www.uoc.edu/web/cat/articles/castells/print.html

Coll, C., \& Monereo, C. (2008). Psicología de la educación virtual. Aprender y enseñar con las tecnologías de la información y la comunicación. Madrid, España: Ediciones Morata S.L.

Coll, C., Mauri, T., \& Onrubia, J. (2008).

Análisis de los usos reales de las TIC en contextos educativos formales: una aproximación socio-cultural. Revista electrónica de investigación educativa. Vol. 10 (1). Obtenido de http://www. scielo.org. $\mathrm{mx} / \mathrm{scielo}$.php?pid=S16070412008000100001\&script=sci_arttext

Coll, C., Onrubia, J., \& Mauri, T. (2007). Tecnología y prácticas pedagógicas: las TIC como instrumentos de mediación de la actividad conjunta de. 38(3), 377-400. Universitat de Barcelona, España.

Erickson, F. (1986). Qualitative Research Methodology for Research in EducationalEnvironments. Handbook of Research on Teaching. Michigan University.

González, D., Bermeo, H. P., \& Ortiz Lugo, G. (2011). Uso de la Web 2.0 como herramienta de apoyo para investigación formativa en entornos universitarios. Caso: Facebook. . México. Obtenido de http://www.virtualeduca.info/fveduca/es/ mexico20111

López de la Madrid, D. (2013). De las interacciones y las tecnologías invisibles. Obtenido de http://pensandoentic.net/pticl sobreluisdavidtobon/

Martínez, G. (2012). Redes sociales y ventaja de su uso educativo. Revista Creatividad y Sociedad. Vol 21. 
Ministerio de Educación Nacional. (2010). Obtenido de $\quad$ http://www.mineducacion.gov.col cvn/1665/w3-article-310158.html

Ministerio de TIC. (s.f.). Computadores para Educar. Obtenido de http://www. computadoresparaeducar.gov.col

Salinas, J. (2004). Innovación docente y uso de las TIC en la enseñanza universitaria.

http://www.mineducacion.gov.co/ cvn/1665/w3-article-310158.html

Stake, R. (1999). Investigación con estudio de casos. Madrid, España: Morata.

UNESCO Institute for Information Technologies (2012). Pedagogies of media and information lietracies.

Yin, R. (1998). El método de casos. Una estrategia de investigación. 47-54. Instituto Tecnológico de Massachusetts. Zapata, M. G. (2014). Ambientes Educativos Cibermultimediales y el Aprendizaje Autónomo. Revista Luciérnaga/Comunicación, Año 6, N12. Págs. 46-53. Disponible en: http:// www.politecnicojic.edu.co/images/downloads/ publicaciones/revista-luciernaga/luciernaga-12/ pdf/4-ambientes_educativos.pdf

\section{Para citar este artículo:}

Gutiérrez, K. (2017). Tic y Educación. Usos de Google Plus en el proceso de enseñanza aprendizaje. Revista Luciérnaga / Comunicación. Año 9, N18. Págs. 41-51.

DOI. 10.33571/revistaluciernaga.v9n18a3

OJS. http://revistas.elpoli.edu.co/index.php/luc/issue/archive

Link. http://www.politecnicojic.edu.co/index.php/revista-luciernaga 